\title{
Surface Brillouin Scattering in Optical Microfibers
}

\author{
Thibaut Sylvestre*, Jean-Charles Beugnot, Joël Cabrel Tchahame, Adrien Godet, Kien Phan Huy, Vincent Laude \\ FEMTO-ST Research Institute, CNRS UMR 6174, University Bourgogne Franche-Comté, Besançon, France \\ *Corresponding author: Thibaut.sylvestre@univ-fcomte.fr
}

\begin{abstract}
By confining light into subwavelength-diameter optical fibers, we demonstrated a new type of Brillouin light scattering from surface and hybrid acoustic waves. This effect could be used to develop new optical sensors that are innovative and highly sensitive.
\end{abstract}

Keywords-Brillouin scattering; tapered optical fibers, photonic crystal fibers, surface acoustic waves.

\section{INTRODUCTION}

Subwavelength-diameter optical fibers, also known as optical fiber micro and nanowires, are the tiny and cousins of standard optical fibers [1,2]. These hair-like slivers of glass, manufactured by tapering optical fibers down to a size hundred times smaller than a strand of hair, have a number of optical and mechanical properties that make them very attractive for both fundamental physics and technological applications. In addition to providing strong light confinement and enhanced nonlinear optical effects, optical microwires also exhibit a large evanescent field, enabling applications not currently possible with comparatively bulky optical fibers. Although microfibers have helped greatly to enhance the optical Kerr effect and stimulated Raman scattering for supercontinuum generation [3], Brillouin scattering in these tiny waveguides has not been explored yet.

Brillouin scattering is one of the most important nonlinear optical processes in optical fibers resulting from the coherent interaction between light and acoustic waves. It has important applications ranging from optical sensors to telecommunications, and it also enables slow and fast light propagation. In standard optical fibers, light excites and interacts with shear and longitudinal bulk elastic waves, giving rise to well-known physical effects such as forward guided acoustic wave Brillouin scattering (GAWBS) and backward stimulated Brillouin scattering (SBS), respectively [4,5].

In a subwavelength-diameter optical fiber, the situation changes dramatically. We report here the experimental observation of Brillouin light scattering from surface and hybrid acoustic waves [6]. To observe the surface waves, we injected a laser beam into a $1-\mu \mathrm{m}$-diameter silica fiber wire. As the beam travels the fiber, it induces infinitesimal vibrations in the wire through the electrostrictive optical force, displacing the surface by a few nanometers. The distortions lead to surface acoustic waves that travel along the wire surface at $3400 \mathrm{~m} \cdot \mathrm{s}^{-1}$. These acoustic phonons in turn affect the light propagation as a part of the light is backscattered with a slight shift of its carrier frequency due to the Doppler effect of in the range 5-6 GHz, far from the standard Brillouin frequency shift of standard optical fibers $(11 \mathrm{GHz})$.

\section{EXPERIMENT}

To produce these silica microwires, colleagues at the Institut d'Optique in Paris heated and stretched standard singlemode optical fibers measuring $125 \mu \mathrm{m}$ in diameter, down to a size of $1 \mu \mathrm{m}$ over $8 \mathrm{~cm}$. Figure 1(a) shows a scheme of the tapered optical fiber drawn from this technique. The remainder of the study was carried out in our lab at the Femto-ST Institute in Besançon. Figure 1(b) shows the experimental setup to detect the acoustic waves. As a pump laser, we used a narrowlinewidth continuous-wave distribute feedback laser (DFB) running at a wavelength of $1,550 \mathrm{~nm}$. The laser output was split into two beams using a fiber coupler. One beam was amplified and injected in the optical microwire through an optical circulator, while the other beam served for detection. We then used a heterodyne detection in which the backscattered light from the microwire was mixed with the input coming from a second fiber coupler. The resulting beat note was then detected using a fast photodiode and Brillouin spectrum recorded using an electrical spectrum analyzer.

\section{a \\ Tapered optical fiber}

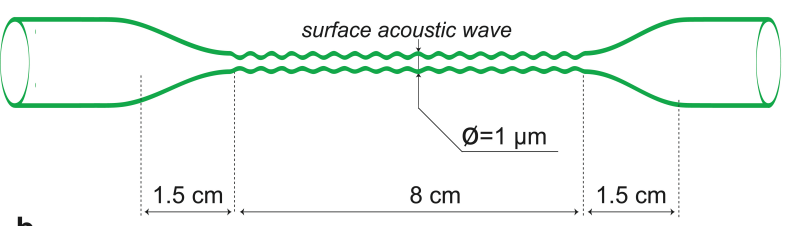

b

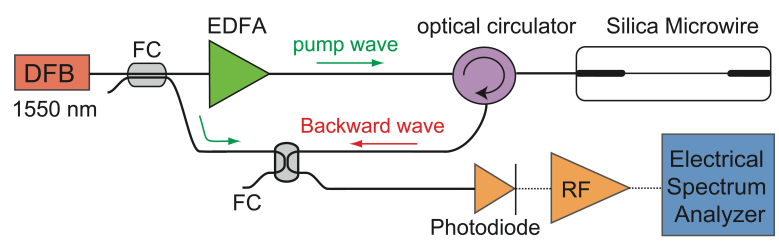

Fig. 1. (a) Scheme of the tapered silica microwire and the surface acoustic wave. (b) Experimental setup. DFB, distributed-feedback laser. FC, 10:90 fibre coupler. EDFA, Erbium-doped fibre amplifier. (c) Experimental spectrum showing the frequency sidebands due to two surface acoustic waves and three hybrid shear-longitudinal waves

\section{RESULTS}

Figure 2 shows the experimental spectrum for an input power of $100 \mathrm{~mW}$. We see the clear emergence of several frequency peaks with different weights in a radio-frequency 
range from 6 to $11 \mathrm{GHz}$. First, the high frequency at 10.86 GHz originates from standard Brillouin scattering in the untapered fiber sections. More importantly, two other peaks appear around $6 \mathrm{GHz}$. We identified them as resulting from Brillouin scattering from surface waves whereas the three other resonances around $9 \mathrm{GHz}$ are clearly the signature of hybrid shear-longitudinal acoustic waves.

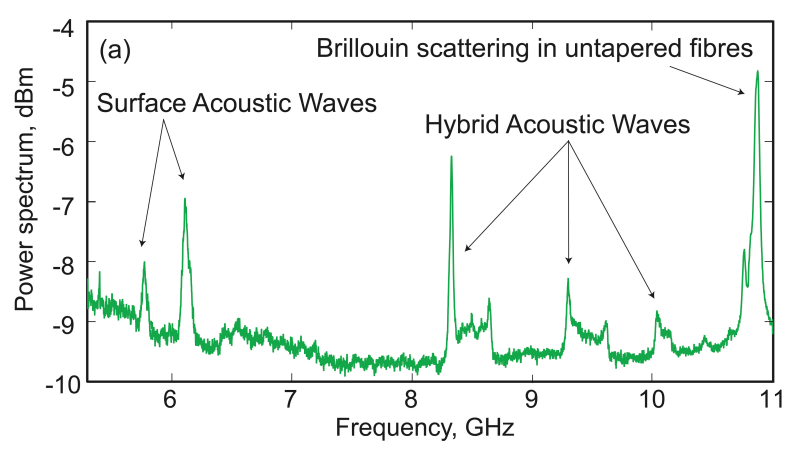

Fig. 2. Experimental beat note radio-frequency spectrum out of a $1 \mu \mathrm{m}$ diameter silica microwire showing the Brillouin sidebands due to two surface acoustic waves and three hybrid shear-longitudinal waves. The highest frequency peak at $10.86 \mathrm{GHz}$ are Brillouin sidebands in untapered fibre sections.

\section{SimUlATIONS}

This observation was subsequently confirmed by a numerical simulation of the elastodynamics equation that includes the electrostrictive optical force [6,7]. Figure 3 shows the computed sound density generated by light in the silica microwire as a function of frequency and wire diameter. As can be seen, we retrieve most of the surface and hybrid acoustic resonances around 6 and $9 \mathrm{GHz}$, as those observed experimentally. Figure 3 also shows that, for a given microwire diameter, multiple hybrid waves with widely spaced frequencies can be simultaneously excited. This means that, in such small subwavelength waveguides, the light-sound interaction is fundamentally different from standard optical fibers. Brillouin spectrum is not simply the signature of a single bulk longitudinal sound wave. In such tiny waveguides, instead, waveguide boundary conditions induce a strong coupling of shear and longitudinal displacements, resulting in a much richer dynamics of light interaction with sound. We can thus the emergence of several anti-crossings in Fig. 3 due to the fact the hybrid waves are not orthogonal and thus strongly interact. The bottom figures show the associated transverse and axial displacements in the microwire for four of surface and hybrid modes. As can be seen, the surface acoustic mode A at $5.382 \mathrm{GHz}$ exhibits a transversal displacement of a few picometres.

\section{CONCLUSIONS}

This type of surface Brillouin scattering had never been observed previously, for it only occurs when light is confined in a subwavelength-diameter waveguide. In standard optical fibers, light travels essentially in the core of the fiber (with a 10-micrometer diameter), and consequently does not generate surface waves. It thus may represent a landmark to foster the investigation of such surface waves in many different photonic platforms, such as photonic crystal fibers, as we recently demonstrated in Refs. [8,9], or in integrated silicon and chalcogenide photonic chips.

Since the waves generated by the confinement of the light travel along the surface, they are sensitive to environmental factors such as temperature, pressure and ambient gas. This makes it possible to design highly sensitive and compact optical sensors for industry. These results also help improve our knowledge of the fundamental interaction between light and sound on an infinitesimally small scale. Surface Brillouin scattering also opens new opportunities in other domains such as nonlinear plasmonics. For instance, one could expect a strong interaction between surface waves and surface plasmons by depositing a thin layer of gold or silver on the microwire.
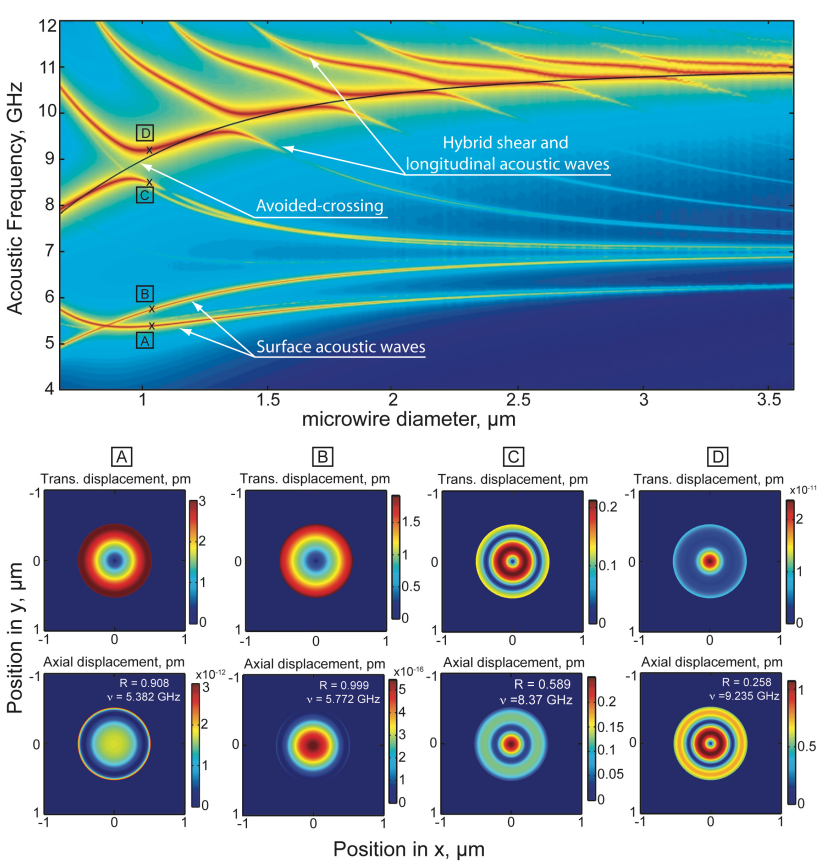

Fig. 3. Numerical simulations of the acoustic wave spectrum and mechanical displacements in a silica microwire generated by light-induced electrostriction. Top : Colour plot of sound density as a function of frequency and for a wire diameter varying from 0.6 to $3.5 \mu \mathrm{m}$. White arrows indicate the surface and hybrid acoustic waves, and the anti-crossing points due to the strong coupling between shear and longitufinal (P) waves. Bottom : Transverse (trans.) and axial displacements in the microwire associated with surface and hybrid acoustic modes labelled A,B,C,D.

\section{ACKNOWLEDGMENTS}

The authors gratefully thank collaborators S. Lebrun and G. Pauliat from the Charles Fabry Laboratory (Institut d'Optique, Orsay, France) for providing the silica microwires, and Raja Hamad and Martin Rochette from McGill University for providing the chalcogenide microwires. These works have been supported by the Agence Nationale de la Recherche (ANR LABEX Action and OASIS projects) and the conseil Régional de Franche-Comté. 


\section{REFERENCES}

[1] L. Tong, et al. Subwavelength-diameter silica wires for low loss optical wave guiding. Nature 426, 816-819 (2003).

[2] G. Brambilla, Optical fiber nanowires and microwires: a review. J. Opt. 12,043001 (2010)

[3] Foster, M. A., Turner, A. C., Lipson, M. \& Gaeta, A. L. Nonlinear optics in photonic nanowires. Opt. Express 16, 1300-1320 (2008).

[4] Shelby, R. M., Levenson, M. \& Bayer, P. Guided acoustic-wave Brillouin scattering. Phys. Rev. B 31, 5244-5252 (1985).

[5] Ippen, E. \& Stolen, R. Stimulated Brillouin scattering in optical fibers. Appl. Phys. Lett. 21, 539-541 (1972).

[6] J.-C. Beugnot, S. Lebrun, G. Pauliat, H. Maillotte, V. Laude and T. Sylvestre. Brillouin light scattering from surface acoustic waves in a subwavelength-diameter optical fiber, Nature Communications 5, 5242, (2014).

[7] J.-C. Beugnot, R. Ahmad, M. Rochette, V. Laude, H. Maillotte, and T. Sylvestre, "Reduction and control of stimulated Brillouin scattering in polymer-coated chalcogenide optical microwires," Optics Letters 39 (3), 482-485 (2014).

[8] Joël Cabrel Tchahame, Jean-Charles Beugnot, Kien Phan Huy, Vincent Laude, Alexandre Kudlinski, and Thibaut Sylvestre, "Surface Brillouin scattering in photonic crystal fibers," Opt. Lett. 266761 (2016).

[9] Joël Cabrel Tchahame, Jean-Charles Beugnot, Alexandre Kudlinski, and Thibaut Sylvestre, "Multimode Brillouin spectrum in a long tapered birefringent photonic crystal fiber," Opt. Lett. 40, 4281-4284 (2015). 